\title{
Ephemeroptera (Insecta) from Pernambuco State, northeastern Brazil
}

\author{
Lucas R. C. Lima ${ }^{1}$, Frederico F. Salles ${ }^{2} \&$ Ulisses Pinheiro
}

\begin{abstract}
${ }^{1}$ Programa de Pós-Graduação em Biologia Animal, Laboratório de Porifera, Centro de Ciências Biológicas, Universidade Federal de Pernambuco, 50670-420 Recife-PE, Brazil. lucaslima_86@hotmail.com

${ }^{2}$ Laboratório de Sistemática e Ecologia de Insetos, Universidade Federal do Espírito Santo, 29933-415 São Mateus-ES, Brazil. ffsalles@gmail.com

${ }^{3}$ Laboratório de Porifera, Centro de Ciências Biológicas, Universidade Federal de Pernambuco, CEP 50670-420 Recife-PE, Brazil. uspinheiro@hotmail.com
\end{abstract}

\begin{abstract}
Ephemeroptera (Insecta) from Pernambuco State, northeastern Brazil. The first list of the species of Ephemeroptera from Pernambuco State, Northeastern Brazil, is presented. The survey was based on collections from numerous rivers and streams of 17 counties. A total of five families, 28 genera, and 38 species were recorded, including 22 genera and 31 species newly recorded from Pernambuco State. Moreover, Farrodes tepui Domínguez, Molineri \& Peters, 1996, Lisetta ernsti Thomas \& Dominique, 2005, and Simothraulopsis (Maculognathus) sabalo Kluge, 2007 are recorded for the first time to Brazil.
\end{abstract}

KEYWORDS. Mayflies; Neotropical; survey; taxonomy.

RESUMO. Ephemeroptera (Insecta) do Estado de Pernambuco, nordeste do Brasil. É apresentada a primeira lista de espécies da ordem Ephemeroptera do Estado de Pernambuco, Região Nordeste do Brasil. O levantamento foi baseado em coletas em vários rios e riachos de 17 municípios. Foram registrados cinco famílias, 28 gêneros e 38 espécies, incluindo 22 novos gêneros e 31 espécies novas para o Estado de Pernambuco. Além disso, Farrodes tepui Domínguez, Molineri \& Peters, 1996, Lisetta ernsti Thomas \& Dominique, 2005 e Simothraulopsis (Maculognathus) sabalo Kluge, 2007 são registrados pela primeira vez para o Brasil.

PALAVRAS-CHAVE. Efemerópteros; levantamento, Neotropical, taxonomia

The taxonomic knowledge about Ephemeroptera in the Northeastern Region can still be considered incipient, especially when compared to the Southern and Southeastern regions of Brazil. Based on the list of species of Ephemeroptera recorded from Brazil (Salles et al. 2004), northeastern Brazil was considered the least studied region of the country with only two species recorded at that time. Much of this divergence was caused by the lack of researchers and faunistic inventories in this region, since only one inventory was made for this region, being restricted to just one family and to one state (see Lima et al. 2010).

According to Salles et al. (2012) there are 256 species recorded from Brazil, distributed in 10 families and 68 genera, with 55 species formally recorded from Northeastern Brazil. Although recent studies have contributed to the knowledge about the distribution of the group in this region (Dias et al. 2008; Lima et al. 2010; Mariano 2010, Salles et al. 2010; Cruz et al. 2011; Boldrini et al. 2012), until this study, only eight species were recorded from the state of Pernambuco: Hermanella froehlichi Ferreira \& Domínguez, 1992, Hermanella maculipennis (Ulmer, 1920), Hydrosmilodon gilliesae Thomas \& Péru, 2004, Hydrosmilodon plagatus Lima, Nascimento \& Salles, 2012, Leentvaaria palpalis Demoulin, 1966, Needhamella ehrhadti (Ulmer, 1920), Traverhyphes (Traverhyphes) frevo Lima, Salles \& Pinheiro, 2011 and Tricorythopsis spongicola Lima, Salles \& Pinheiro, 2011 (Lima et al. 2011, 2012).

In this paper we provide the first inventory of Ephemeroptera from the State of Pernambuco, as well as new records from the Northeastern Region and Brazil.

\section{MATERIAL AND METHODS}

The State of Pernambuco is located in the Northeastern Region of Brazil and has an area of $98.311 \mathrm{~km}^{2}$. The state is divided into three regions according to the physical environment: Zona da Mata (11\%), located on the eastern part of the state and characterized by humid climate and Atlantic Forest vegetation; Agreste (19\%), a transition zone dominated by semi-arid conditions; and Sertão (70\%), centralwestern part of the state and characterized by semi-arid climate and caatinga vegetation (Carvalho 2008).

The material was collected in 34 localities of 17 municipalities located at Zona da Mata (Fig. 1), between March/ 2009 and February/2011. Nymphs were collected in several submerged substrates (stones, gravels, bedrocks, macrophytes, leafs, woody debris) of each environment by manually picking the specimens or using a D-net. Adults were caught on light traps right after sunset.

Dissected parts of the specimens studied were mounted on microscope slides using Canada Balsam as mounting media. All material was preserved in ethanol $80 \%$. The identifications were based in Dominguez et al. (2006) and Salles (2006), aided by pertaining papers to each taxon. For information about the geographical distribution of species were consulted Domínguez et al. (2006) and the list of species of Ephemeroptera recorded from Brazil on the website "Ephemeroptera do Brazil" updated frequently (Salles et al. 2012).

To analyze the records of the species in river basins, we adopted the classification of the Agência Nacional de Águas. 


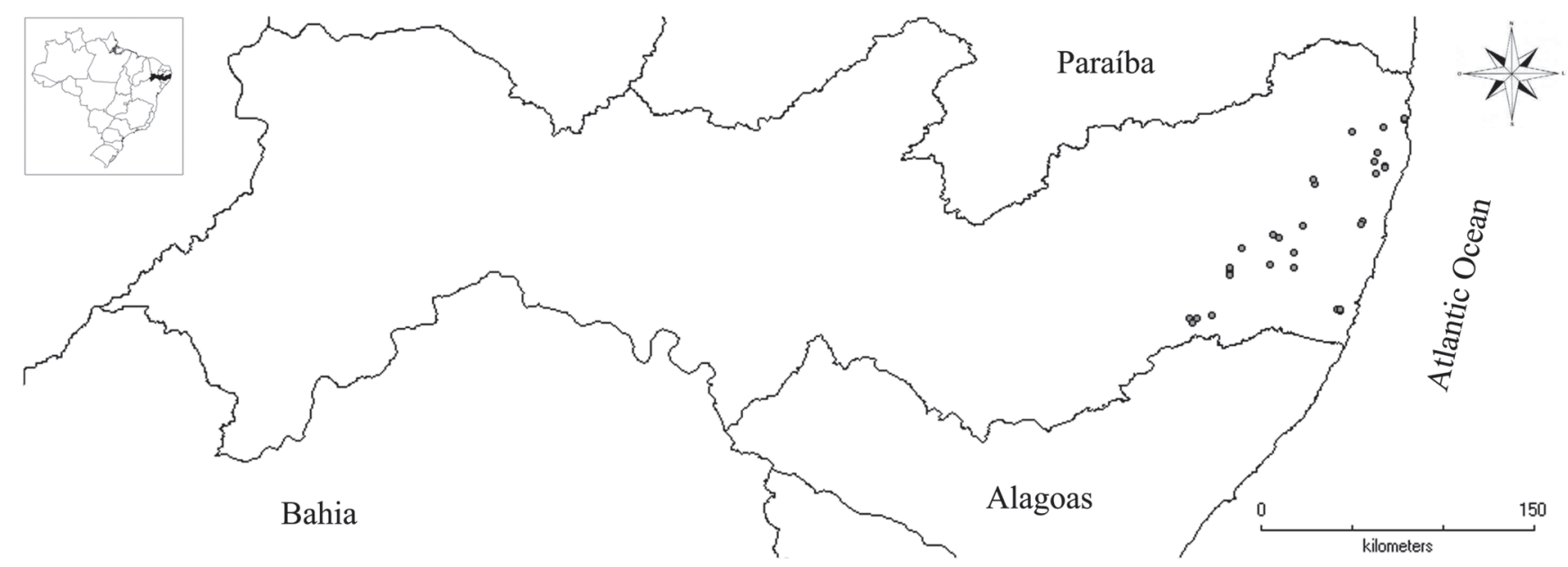

Fig. 1. Study area showing the map of Pernambuco State with the respective collecting localities.

Records of Brazilian states follow official state abbreviations. The material is deposited in the following institutions: Coleção Zoológica Norte Capixaba (CZNC) and Coleção Entomológica da Universidade Federal de Pernambuco (UFPE).

\section{RESULTS AND DISCUSSION}

Five families were found in this study (Baetidae, Caenidae, Leptohyphidae, Leptophlebiidae and Polymitarcyidae) distributed among 28 genera and 38 species. In this total 22 genera and 31 species are reported for the first time from the state, whereas the first record of Farrodes tepui Domínguez, Molineri \& Peters, 1996, Lisetta ernsti Thomas \& Dominique, 2005, and Simothraulopsis (Maculognathus) sabalo Kluge, 2007 are presented for Brazil. These results increased from 55 to 66 the number of species recorded from the Northeastern Region. Below we provide a list of all mayfly species recorded for the state. For each species listed data on geographical distribution are provided, and comments are made when necessary.

\section{List of species \\ Baetidae}

\section{Americabaetis alphus Lugo-Ortiz \& McCafferty, 1996}

Previous distribution. BRAZIL: Amazon Basin (AM, RR), Araguaia-Tocantins Basin (MT), Atlântico Leste Basin (BA, SE), Atlântico Nordeste Oriental Basin (AL), Atlântico Sudeste Basin (ES, RJ, SP), Atlântico Sul Basin (RS), Paraná River Basin (GO, MG, PR, SP), Parnaíba River Basin (PI), and Uruguai River Basin (SC). ARGENTINA, BOLIVIA, CHILE, and PARAGUAY.

Material. PERNAMBUCO: Amaraji (Rio Amaraji próximo à Usina Amaraji, 8`21'48.9”S 35²8'49.0”W, 320m), 12 nymphs, 20.I.2011, L.R.C. Lima, G. Nicacio, R.P. Salomão cols. (UFPE); Amaraji, (Rio Amaraji, Cachoeira do Rio Morto, 8²0'59.1'S 3530'21.0”W), 1 nymph, 363m, 20.I.2011, L.R.C. Lima, G. Nicacio, R.P. Salomão cols. (UFPE); Bonito (Rio Verde, Camping Bonito Ecoparque, 8`31'58.6”S 3543’26.3”W), 28/ i/2010, 3 nymphs, Lima L.R.C. Lima, G. Nicacio, cols. (CZNC); Cabo de
Santo Agostinho (Rio Pirapama, 8¹6'58.1"S 3503'46.6”W, 30m), 8 nymphs, 18.VIII.2010, L.R.C. Lima, col. (CZNC); Camaragibe, (Rio Besouro, 759'06.4”S 3500'13.9”W, 66m), 3 nymphs, 02.VII.2009, L.R.C. Lima coll. (UFPE); same as preceding except 3 nymphs, 2 o" (light trap), 1 ㅇ (light trap), 08.VII.2009 (UFPE); Jaboatão dos Guararapes, (Rio Capibaribe, próximo a Transpetro, 802'47.4”S 3459'41.9”W, 51m), 7 nymphs, 24.X.2009, L.R.C. Lima, G. Nicacio, cols. (CZNC); Primavera, (Cachoeira do Urubu, 8¹8'12.6”'S 35²1'21.9”W), 23 nymphs, 31.X.2009, L.R.C. Lima, C.C. Nobre cols. (CZNC); Recife (Parque Dois Irmãos, Açude do Prata, $8^{\circ} 00^{\prime 2} 28.5^{\prime}$ 'S 3456’54.3”W), 7 nymphs, 12.IX.2009, L.R.C. Lima, G. Nicacio cols. (CZNC); Ribeirão, (Rio Amaraji, Cachoeira do Amor,

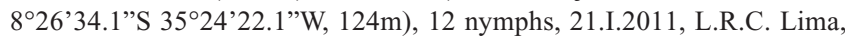
G. Nicacio, R.P. Salomão cols. (UFPE); Rio Formoso, (Fazenda São Manuel,

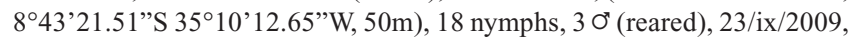
L.R.C. Lima coll. (UFPE); same as preceding except 6 nymphs, 04.V.2010 (UFPE); São Benedito do Sul (Cachoeira Poço do Caboclo, 845'53.4”S 3555'16.1'W, 532m), 1 ơ (light trap), 13.XII.2009, L.R.C. Lima, G. Nicacio cols. (UFPE); Tamandaré, (Reserva Biológica de Saltinho, Rio Mamucabas, 843'52.7'S 35¹0'24.8'W, 38m), 5 nymphs, 23.IX.2009, L.R.C. Lima coll. (CZNC); same as preceding except $3 \mathrm{O}^{\prime \prime}$ (light trap), (Córrego da Sede, 8`43'53.7'’S 35¹0'25.8”W) (UFPE); Vitória de Santo Antão, (Fazenda Esperança, 85'59.51'S 35¹7'49.6”W), 34 nymphs, 09.VIII.2009, L.R.C. Lima, G. Nicacio, R.P. Salomão (CZNC); same as preceding except 10 nymphs, 2 ơ (light trap), 2 \% (light trap), 30.VIII.2009 (CZNC).

\section{Americabaetis labiosus Lugo-Ortiz \& McCafferty, 1996}

Previous distribution. BRAZIL: Atlântico Leste Basin (BA), Atlântico Sudeste Basin (ES, RJ, SP), Atlântico Sul Basin (RS), Paraná River Basin (MG, PR, SP), and Uruguai River Basin (SC). PARAGUAY and URUGUAY.

Material. PERNAMBUCO: Amaraji, (Rio Amaraji próximo a Usina Amaraji, $8^{\circ} 21^{\prime} 48.9^{\prime}$ 'S $35^{\circ} 28^{\prime} 49.0^{\prime \prime} \mathrm{W}, 320 \mathrm{~m}$ ), 1 nymph, 2 ᄋ (reared), 20.I.2011, L.R.C. Lima, G. Nicacio, R.P. Salomão cols. (UFPE); Amaraji (Cachoeira do Rio Morto, $8^{\circ} 20^{\prime} 59.1^{\prime \prime S} 35^{\circ} 30^{\prime} 21.0^{\prime \prime} \mathrm{W}, 363 \mathrm{~m}$ ), 1 nymph, 20.I.2011, L.R.C. Lima, G. Nicacio, R.P. Salomão cols (UFPE); Ribeirão,

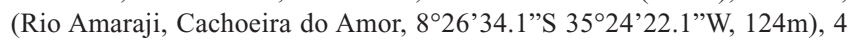
nymphs, 21.I.2011, L.R.C. Lima, G. Nicacio, R.P. Salomão cols. (UFPE); Tamandaré, (Reserva Biológica de Saltinho, Rio Mamucabas, 843'21.6”'S $35^{\circ} 11^{\prime} 14^{\prime \prime}$ 'W, 90m,), 5 nymphs, $10^{\prime \prime}$ (reared), 22.IX.2009, L.R.C. Lima coll. (CZNC); same as preceding except 17 nymphs, $3 O^{\circ}$ (light trap), 24.IX.2009 (CZNC). 


\section{Apobaetis fiuzai Salles \& Lugo-Ortiz, 2002}

Previous distribution. BRAZIL: Amazon Basin (RR), Atlântico Leste Basin (BA) Atlântico Sudeste Basin (ES, RJ, SP), Paraguai River Basin (MT), Parnaíba River Basin (MA), Paraná River Basin (MG), and São Francisco River Basin (MG).

Material. PERNAMBUCO: São Benedito do Sul (Cachoeira Poço do Caboclo, $8^{\circ} 45^{\prime} 53.4^{\prime \prime}$ ' $35^{\circ} 55^{\prime} 16.1$ 'W, 532m), 1 nymph, 23.II.2010, L.R.C. Lima, G. Nicacio, U.S. Pinheiro cols. (UFPE).

\section{Aturbina beatrixae Gillies, 2001}

Previous distribution. BRAZIL: Atlântico Leste Basin (BA), Atlântico Nordeste Oriental (RN), Atlântico Sudeste Basin (ES, MG), Paraná River Basin (PR, SP), and Uruguai River Basin (SC). ARGENTINA and URUGUAY.

Material. PERNAMBUCO: Camaragibe, (Rio Besouro, 759'06.4”S $35^{\circ} 00^{\prime} 13.9^{\prime \prime} \mathrm{W}, 66 \mathrm{~m}$ ), 1 nymph, 2 \% (light trap), 02.VII.2009, L.R.C. Lima coll. (UFPE); same as preceding except 1 nymph, 2 (light trap), 10 (light trap), 08.VII.2009 (CZNC); same as preceding except 3 \% (light trap), 5 O $^{7}$ (light trap), 28.II.2010 (CZNC); Ribeirão, (Cachoeira do Amor, 8²6’34.1”S $35^{\circ} 24^{\prime} 22.1$ 'W, 124 m), 2 nymphs, 21.I.2011, L.R.C. Lima, G. Nicacio, R.P. Salomão cols. (UFPE); São Benedito do Sul, (Cachoeira Poço do Caboclo, 845'53.4”'S 3555'16.1”'W, 532 m), 2 nymphs, 26.II.2011, L.R.C. Lima, F.F. Salles cols. (UFPE); Vitória de Santo Antão, (Fazenda Esperança, $\left.8^{\circ} 5^{\prime} 59.51^{\prime \prime} \mathrm{S} 35^{\circ} 17^{\prime} 49.6^{\prime \prime} \mathrm{W}\right), 20^{\prime}$ (light trap), 2 Q (light trap), 30.VIII.2009, L.R.C. Lima, G. Nicacio, R.P. Salomão cols. (UFPE).

\section{Baetodes santatereza Salles \& Polegatto, 2008}

Previous distribution. BRAZIL: Paraná River Basin (SP).

Material. PERNAMBUCO: Bonito, (Cachoeira do Engenho Barra Azul, $\left.8^{\circ} 30^{\prime} 39.3^{\prime \prime S} 35^{\circ} 43^{\prime 2} 21.0^{\prime \prime} \mathrm{W}\right), 2$ nymphs, 28.I.2010, L.R.C. Lima, G. Nicacio cols. (CZNC); Bonito, (Cachoeira Véu da Noiva II, $8^{\circ} 32^{\prime} 51.3^{\prime}$ 'S, 35'43'30.7'W), 3 nymphs, 29.I.2010, L.R.C. Lima, G. Nicacio cols. (CZNC); Bonito, (Rio Verde, Camping Bonito Ecoparque, 8³1'58.6”S $35^{\circ} 43^{\prime} 26.3^{\prime}$ 'W), 1 nymph, 27.I.2010, L.R.C. Lima, G. Nicacio cols. (CZNC); Cabo de Santo Agostinho, (Rio Pirapama, $8^{\circ} 16^{\prime} 58.1$ "S $\left.35^{\circ} 03^{\prime} 46.6^{\prime \prime} \mathrm{W}, 30 \mathrm{~m}\right), 6$ nymphs, 18.VIII.2010, L.R.C. Lima, cols. (UFPE); same as preceding except 37 nymphs, Rio Pavão (CZNC); Jaqueira, Rio Pirangi, PE-126 próximo a ponte ( $\left.8^{\circ} 44^{\prime} 53^{\prime \prime} \mathrm{S} 35^{\circ} 48^{\prime} 51.1^{\prime \prime} \mathrm{W}, 189 \mathrm{~m}\right), 13$ nymphs, 12.XII.2009, L.R.C. Lima, G. Nicacio cols. (CZNC); same as preceding except 5 nymphs, 17.IV.2010 (CZNC); Primavera, (Riacho da Bica, estrada para a Cachoeira do Urubu, $8^{\circ} 18^{\prime} 30.7^{\prime \prime}$ 'S $\left.35^{\circ} 21^{\prime} 27.8^{\prime \prime} \mathrm{W}, 162 \mathrm{~m}\right), 8$ nymphs, 31.X.2009, L.R.C. Lima, C.C. Nobre cols. (UFPE); São Benedito do Sul, (Cachoeira Poço do Soldado, S845'52.9”, 3553'06.1”W, 321m), 2 nymphs, 12.XII.2009, L.R.C. Lima, G. Nicacio cols. (CZNC); São Benedito do Sul, (Poço do Caboclo, 845'53.4”S 3555'16.1”'W, 532m), 3 nymphs, 28.VIII.2010 L.R.C. Lima, G. Nicacio cols (UFPE); São Benedito

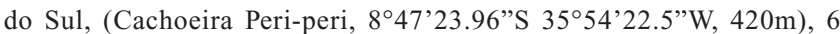
nymphs, 13.XII.2009, L.R.C. Lima, G. Nicacio cols. (CZNC); Vitória de Santo Antão, (Bica do Oiteiro, $8^{\circ} 4$ '42.6”'S, 35 $18^{\prime} 16.4 \mathrm{~W}$ ), 1 nymph, 09.VIII.2009, L.R.C. Lima, G. Nicacio, R.P. Salomão cols. (UFPE).

\section{Callibaetis gonzalezi Navás, 1934}

Previous distribution. BRAZIL: Atlântico Leste Basin (BA). ARGENTINA, BOLIVIA, and PARAGUAY.

Material. PERNAMBUCO: Rio Formoso, (Fazenda São Manuel, $\left.8^{\circ} 43^{\prime} 21.51^{\prime} \mathrm{S} 35^{\circ} 10^{\prime} 12.65^{\prime \prime} \mathrm{W}, 50 \mathrm{~m}\right), 1$ nymph, 23.IX.2009, L.R.C. Lima coll. (UFPE); Vitória de Santo Antão, (Fazenda Esperança, 85'59.51”'S $35^{\circ} 17^{\prime} 49.6$ ”W), 5 nymphs, 1 ○ (reared), 30.VIII.2009, L.R.C. Lima, G. Nicacio, R.P. Salomão cols. (UFPE);

\section{Callibaetis guttatus Navás, 1920}

Previous distribution. BRAZIL: Atlântico Sudeste Basin (ES, RJ). ARGENTINA.

Material. PERNAMBUCO: Araçoiaba, (Açude Campo Grande, 7'50'13.3”'S 356’39.6W, 133m), 3 nymphs, $10^{\circ}$ (reared), 07.XI.2009, L.R.C. Lima, G. Nicacio cols. (UFPE); same as preceding except 2 nymphs, 1 ᄋ (reared), $20^{\prime \prime}$ (reared), 26.XI.2010 (UFPE); Itamaracá (brejo da estrada para a Lagoa Azul, 746’41.66”'S 3451’3.74”'W, 32m), 6 nymphs, 1 \% (reared), 24.V.2009, L.R.C. Lima coll. (UFPE); same as preceding except 3 nymphs, 1 O' (reared), 31.V.2009 (UFPE); Recife, (Açude do Prata, 800'28.5”'S $34^{\circ} 56^{\prime} 54.3$ ”'W, 31m), 1 \% (reared), 12.IX.2009, L.R.C. Lima coll. (UFPE).

\section{Callibaetis pollens Needham \& Murphy, 1924}

Previous distribution. BRAZIL: Atlântico Leste Basin (BA), (GO), Atlântico Nordeste Ocidental Basin (MA), Atlântico Nordeste Oriental Basin (CE), Parnaíba River Basin (PI), Paraguay River Basin (MS), and São Francisco River Basin (BA, GO). ARGENTINA.

Material. PERNAMBUCO: Ribeirão, (Rio Amaraji, Cachoeira do Amor, 8²6'34.1'S 35²4'22.1' 'W, 124m), 1 nymph, 1 우 (reared), 1 O' (reared), 21.I.2011, L.R.C. Lima, G. Nicacio, R.P. Salomão cols. (UFPE); Rio Formoso (Fazenda São Manuel, 843'21.51'S 35¹0'12.65'W, 50m), 2 nymphs, $10^{\prime \prime}$ (reared), 05.V.2010, L.R.C. Lima coll. (UFPE); São Benedito do Sul, (Cachoeira Poço do Caboclo, 845'53.4”'S 3555'16.1”W, $532 \mathrm{~m}$ ), 1 nymph, 26.II.2011, L.R.C. Lima, F.F. Salles cols. (UFPE) São Benedito do Sul (Cachoeira Poço do Soldado, 845'52.9”'S 3553'06.1”W, $321 \mathrm{~m}$ ), 4 nymphs, 23.II.2010, L.R.C. Lima, G. Nicacio cols. (UFPE).

\section{Camelobaetidius billi Thomas \& Dominique, 2001}

Previous distribution. BRAZIL: Atlântico Leste Basin (BA), Atlântico Sudeste Basin (ES),Amazon Basin (AM), and São Francisco River Basin. FRENCH GUIANA.

Material. PERNAMBUCO: Amaraji, (Rio Amaraji próximo a usina Amaraji, $8^{\circ} 21^{\prime} 48.9^{\prime}$ 'S 35²8'49.0”W, 320m), 2 nymphs, 20.I.2011, L.R.C. Lima, G. Nicacio, R.P. Salomão cols. (UFPE); Amaraji, (Cachoeira do Rio Morto, 8²0'59.1'S 35³0'21.0”W, 363m), 2 nymphs, 20.I.2011, L.R.C. Lima, G. Nicacio, R.P. Salomão cols. (UFPE); Bonito, (Rio Verde, Camping Bonito Ecoparque, 8`31'58.6”S 3543'26.3”W), 8 nymphs, 27.I.2010, L.R.C. Lima, G. Nicacio cols. (UFPE); Bonito, (Rio Verde, Camping Acampamento do Mágico, 8³0'46.8'S 3543'14’W), 6 nymphs, 28.I.2010, L.R.C. Lima, G. Nicacio cols. (UFPE); Bonito, (Cachoeira do Engenho Barra Azul, 8`30’39.3”S 35॰43’21.0”W), 7 nymphs, 28.I.2010, L.R.C. Lima, G. Nicacio cols. (UFPE); Bonito, (Cachoeira Véu da Noiva II, 8³2'51.3”S 35'43'30.7’W), 27 nymphs, $10^{\prime \prime}$ (reared), 29.I.2010, L.R.C. Lima, G. Nicacio cols. (UFPE); Cabo de Santo Agostinho, (Rio Pavão, 8¹7'51.5"S 3503'57.1'W, 58m), 1 nymph, 18.VIII.2010, L.R.C. Lima cols. (UFPE); Camaragibe, (Rio Besouro, 759'06.4'S, 3500'13.9'W, 66m), 23 nymphs, 21.VI.2009, L.R.C. Lima coll. (CZNC); same as preceding except 8 nymphs, 1 o (reared), 02.VII.2009 (UFPE); same as preceding except 5 nymphs, 08.IX.2009 (UFPE); Jaqueira, (Rio Pirangi, PE-126 próximo a ponte, 844'53”'S 3548'51.1”W, 189m), 18 nymphs, 12.XII.2009, L.R.C. Lima, G. Nicacio cols. (UFPE); Primavera, (Cachoeira do Urubu, 8¹8'12.6”S $\left.35^{\circ} 21^{\prime} 21.9^{\prime \prime W}\right), 27$ nymphs, 31.X.2009, L.R.C. Lima C.C. Nobre cols. 
(UFPE); same as preceding except 4 nymphs, 28.VIII.2010 (UFPE); Ribeirão, (Rio Amaraji, Cachoeira do Amor, 8 $\left.8^{\circ} 26^{\prime} 34.1^{\prime \prime} \mathrm{S}, 35^{\circ} 24^{\prime} 22.1^{\prime \prime} \mathrm{W}, 124 \mathrm{~m}\right), 2$ nymphs, 21.I.2011, L.R.C. Lima, G. Nicacio, R.P. Salomão cols. (UFPE); São Benedito do Sul, (Cachoeira Poço do Caboclo, 845'53.4”'S 3555'16.1W, 532m), 6 nymphs, 13.XII./2009, L.R.C. Lima, G. Nicacio cols. (UFPE); São Benedito do Sul, (Cachoeira Poço do Soldado, 845'52.9'’S 3553'06.1'W, $321 \mathrm{~m}$ ), 5 nymphs, 12.XII.2009, L.R.C Lima, G. Nicacio cols. (UFPE); Tamandaré, (Reserva Biológica de Saltinho, Rio Mamucabas próximo ao açude, 843'21.6”'S 35¹1'14'W, 90m), 5 nymphs, 24.IX.2009, L.R.C. Lima coll. (CZNC); Vitória de Santo Antão, (Bica do Oiteiro, 84'42.6"S 35¹8'16.4W), 14 nymphs, 09.VIII.2009 L.R.C. Lima, G. Nicacio, R.P. cols. (UFPE); same as preceding except 1 nymph, 30.VIII.2009 (UFPE).

\section{Camelobaetidius cayumba (Traver \& Edmunds, 1968)}

Previous distribution. BRAZIL: Amazon Basin (RR, RO), Araguaia-Tocantins Basin (GO), Atlântico Nordeste Ocidental Basin (MA), Atlântico Nordeste Oriental Basin (CE), and Parnaíba River Basin (PI). COLOMBIA, FRENCH GUIANA, and PERU.

Material. PERNAMBUCO: Amaraji, (Rio Amaraji próximo a usina Amaraji, 8²1'48.9'S 35²8'49.0'W, 320m), 1 nymph, 20.I.2011, L.R.C. Lima, G. Nicacio, R.P. Salomão cols. (UFPE); Amaraji, (Cachoeira do Rio Morto, 8`20'59.1'S 35³0'21.0”'W, 363m), 1 nymph, 20.I.2011, L.R.C. Lima, G. Nicacio, R.P. Salomão cols. (UFPE); Barra de Guabiraba, (Cachoeira do Galo, 8²5'05.5”S 35³9'48.2”W, 502m), 3 nymphs, 20.III.2011, L.R.C. Lima, G. Nicacio cols. (UFPE)Cabo de Santo Agostinho, (Rio Pirapama, $8^{\circ} 16$ '58.1'S 3503'46.6”W, 30m), 1 nymph, 18.VIII.2010, L.R.C. Lima, coll. (UFPE); same as preceding except Rio Pavão, 1 nymph (UFPE); Camaragibe, (Rio Besouro, 759'06.4”S 3500'13.9”W, 66m), 1 \% (reared), 02.VII.2009, L.R.C. Lima coll. (CZNC); same as preceding 1 nymph, 08.VII.2009 (CZNC); Cortês, (Banho da Ilha, 8`30'06.5”S 35³1'28.6”W, 223m), 10 nymphs, 20.III.2011, L.R.C. Lima, G. Nicacio cols. (UFPE); Jaboatão dos Guararapes, (Rio Capibaribe próximo a Transpetro, 802'47.4'S 3459'41.9'W, $51 \mathrm{~m}$ ), 9 nymphs, 24.X.2009 (CZNC); same as preceding except 2 nymphs, 08.IV.2010 (CZNC); Jaqueira, (Rio Pirangi, PE-126 próximo a ponte, 844'53”S 3548'51.1"W, 189m), 3 nymphs, 12.XII.2009, L.R.C. Lima, G. Nicacio cols. (UFPE); 3 nymphs, same as preceding except 17.IV.2010 (UFPE); Primavera, (Cachoeira do Urubu, 8¹8'12.6”S 35²1'21.9”W), 2 nymphs, 31.X.2009, L.R.C. Lima, C.C. Nobre cols. (UFPE); Ribeirão, (Rio Amaraji, Cachoeira do Amor, 8²6'34.1"S 35²4'22.1'W, 124m), 14 nymphs, 1 ㅇ (reared), 21.I.2011, L.R.C. Lima, G. Nicacio, R.P. Salomão cols. (UFPE); Ribeirão, (Rio Amaraji próximo ao Engenho Bastiões, 8³0'49.9”S 35²4’02.2”W, 114m), 28 nymphs, 05.II.2011, L.R.C. Lima, G. Nicacio cols. (UFPE).

Remarks. The nymphs of $C$. cayumba showed a large variation in the body length $(3.6-6.2 \mathrm{~mm})$ in which differ the original description by Traver \& Edmunds (1968) and the redescription made by Thomas et al. (2001), in which nymphs showed $3.5 \mathrm{~mm}$ body length. The distinct pattern of tergal markings also varied, being slightly distinguishable or absent in some individuals. Due the shape of the mouthparts, the number of the tarsal claw denticles, among other characteristics was possible to identify the species.

\section{Camelobaetidius francischettii Salles, Andrade \& Da-Silva, 2005}

Previous distribution. BRAZIL: Atlântico Leste Basin (BA), Atlântico Nordeste Oriental Basin (AL), and Atlântico Sudeste Basin (RJ, ES).
Material. PERNAMBUCO: Amaraji, (Cachoeira do Rio Morto, $8^{\circ} 20^{\prime} 59.1$ ”S 35³0'21.0”W, 363m), 3 nymphs, 20.I.2011, L.R.C. Lima, G. Nicacio, R.P. Salomão cols. (UFPE); Bonito, (Camping Bonito Ecoparque, 8`31'58.6”S 3543'26.3”W), 2 nymphs, 27.I.2010, L.R.C. Lima, G. Nicacio cols. (UFPE); Bonito, (Camping Acampamento do Mágico 8³0’46.8”S 3543'14'W), 7 nymphs, 28.I.2010, L.R.C. Lima, G. Nicacio cols. (CZNC); Bonito, (Cachoeira do Engenho Barra Azul, 8³0'39.3”S 3543’21.0”W), 6 nymphs, 28.I.2010, L.R.C. Lima, G. Nicacio cols. (UFPE); Bonito, (Cachoeira Véu da Noiva II, 8³2'51.3”S 3543'30.7'W), 3 nymphs, 29.I.2010, L.R.C. Lima, G. Nicacio cols. (UFPE); São Benedito do Sul, (Cachoeira Peri-peri, 847'23.96”'S 3554'22.5”W, 420m), 10 (reared), 10" (reared), 13.XII.2009, L.R.C. Lima, G. Nicacio cols. (UFPE); São Benedito do Sul, (Cachoeira Poço do Soldado, 845'52.9”S 3553'06.1”W, 321m), 3 nymphs, 12.XII.2009, L.R.C. Lima, G. Nicacio cols. (UFPE).

\section{Camelobaetidius lassance Salles \& Serrão, 2005}

Previous distribution. Brazil: Atlântico Leste Basin (BA), Paraná River Basin (SP), and São Francisco River Basin (MG).

Material. PERNAMBUCO: Barra de Guabiraba, (Cachoeira do Galo, $8^{\circ} 25^{\prime} 05.5^{\prime} \mathrm{S} 35^{\circ} 39^{\prime} 48.2^{\prime \prime} \mathrm{W}, 502 \mathrm{~m}$ ), 1 nymph, 20.III.2011, L.R.C. Lima, G. Nicacio cols. (UFPE); Cortês, (Banho da Ilha, 8³0'06.5"S 35³1'28.6”W, 223m), 1 \% (reared), 05.II.2011, L.R.C. Lima, G. Nicacio cols. (UFPE); same as preceding except 5 nymphs, 20.III.2011 (UFPE).

\section{Cloeodes hydation McCafferty \& Lugo-Ortiz, 1995}

Previous distribution. BRAZIL: Araguaia-Tocantins Basin (MT), Atlântico Sudeste Basin (ES), and São Francisco River Basin (MG).

Material. PERNAMBUCO: Primavera, (Cachoeira do Urubu, $\left.8^{\circ} 18^{\prime} 12.6^{\prime \prime S} 35^{\circ} 21^{\prime} 21.9^{\prime \prime} \mathrm{W}\right), 3$ nymphs, 31.X.2009, L.R.C., C.C. Nobre cols. (UFPE); Vitória de Santo Antão, (Fazenda Esperança, $8^{\circ} 5^{\prime} 59.51$ "'S $\left.35^{\circ} 17^{\prime} 49.6^{\prime} \mathrm{W}\right), 2$ nymphs, 09.VIII.2010, L.R.C. Lima, G. Nicacio, R.P. Salomão cols. (UFPE).

\section{Cloeodes irvingi Waltz \& McCafferty, 1987}

Previous distribution. BRAZIL: Atlântico Nordeste Oriental Basin (CE), Araguaia-Tocantins Basin (MT), Atlântico Sudeste Basin (ES), and São Francisco River Basin (GO, MG). PARAGUAY.

Material. PERNAMBUCO: São Benedito do Sul, (Cachoeira Poço do Caboclo, 8`45'53.4”S 3555'16.1”W), 1 nymph, 13.XII.2009, L.R.C. Lima, G. Nicacio (UFPE); São Benedito do Sul, (Cachoeira Poço do Soldado, 845'52.9'S 3553'06.1'W), 4 nymphs, 12.XII.2009, L.R.C. Lima, G. Nicacio (UFPE).

\section{Cloeodes sp.}

Material. PERNAMBUCO: Rio Formoso, (Riacho da Gameleira, Reserva Biológica de Saltinho, 8`43'12.3”S 35¹0'32.9'W, 47m), 6 nymphs, 24.IX.2009, L.R.C. Lima col. (CZNC); Tamandaré, (Rio Mamucabas, Reserva Biológica de Saltinho, 843'52.7'S 3510'24.8”'W, 38 m), 1 O' (reared), 21.IX.2009, L.R.C. Lima col. (CZNC); same as preceding except 21.IX.2009, Córrego da Sede, 1 o (reared), (CZNC); same as preceding except 22.IX.2009, 1 nymph, 1 ㅇ (reared) (CZNC); same as preceding except 2 nymphs, 2 (reared), 13.V.2010 (UFPE); same as preceding except 
1 Ơ (reared), 14.V.2010 (CZNC); same as preceding except 1 Ơ (reared), 08.VIII.2010 (CZNC).

Remarks. Cloeodes sp. is a new species and its description will be submitted in another study.

\section{Cryptonympha dasilvai Salles \& Francischetti, 2004}

Previous distribution. BRAZIL: Atlântico Leste Basin (BA), Atlântico Sudeste Basin (ES, RJ, SP), and Paraná River Basin (SP).

Material. PERNAMBUCO: Camaragibe, (riacho de $1^{\circ}$ ordem, $\left.7^{\circ} 56^{\prime} 28.01^{\prime \prime S} 34^{\circ} 59^{\prime} 7.32^{\prime \prime} \mathrm{W}\right), 5$ nymphs, 28.II.2010, L.R.C. Lima coll. (UFPE); Igarassu, (Refúgio Charles Darwin, Riacho Jacocá, $7^{\circ} 48^{\prime} 57.5^{\prime}$ ”S $34^{\circ} 57^{\prime} 19.1$ ”'W, 37m), 11 nymphs 22.II.2010, L.R.C. Lima coll. (UFPE); same as preceding except 3 nymphs, 05.III.2010 (UFPE); Ribeirão (Cachoeira do Amor, $8^{\circ} 26^{\prime} 34.1^{\prime \prime S} 35^{\circ} 24^{\prime} 22.1^{\prime \prime} \mathrm{W}, 124 \mathrm{~m}$ ), 2 nymphs, 21.I.2011, L.R.C. Lima, G. Nicacio, R.P. Salomão cols. (UFPE); São Benedito do Sul, (Cachoeira Poço do Caboclo, 845'53.4”S 35'55'16.1”'W, 532 m), 4 nymphs, 13.XII.2009, L.R.C. Lima, G. Nicacio cols. (UFPE).

\section{Harpagobaetis gulosus Mol, 1986}

Previous distribution. BRAZIL: Amazon Basin (RR) and Araguaia-Tocantins Basin (GO, MA, MT). SURINAME.

Material. PERNAMBUCO: Amaraji, (Rio Amaraji próximo a Usina Amaraji, 8²1'48.9”S, 35²8'49.0”W, 320m), 1 nymph, 20.I.2011, L.R.C. Lima, G. Nicacio, R.P. Salomão cols. (UFPE); Jaqueira, (Rio Pirangi, PE126 próximo a ponte, $8^{\circ} 44^{\prime} 53^{\prime} \mathrm{S} 35^{\circ} 48^{\prime} 51.1$ ”W, $\left.189 \mathrm{~m}\right), 1$ nymph, 17.IV.2010, L.R.C. Lima, G. Nicacio (UFPE); Ribeirão, (Cachoeira do Amor, 8²6’34.1”S 35²4'22.1'W, 124 m), 4 nymphs, 21.I.2011, L.R.C. Lima, G. Nicacio, R.P. Salomão cols (UFPE).

\section{Paracloeodes sp.}

Material. PERNAMBUCO: Ribeirão (Cachoeira do amor, $8^{\circ} 26^{\prime} 34.1^{\prime \prime} \mathrm{S}$ $35^{\circ} 24^{\prime} 22.1$ ”W, 124m), 2 nymphs, 1 \% (reared), 21.I.2011, L.R.C. Lima, G. Nicacio, R.P. Salomão cols (UFPE).

Remarks. Based on nymphs, Paracloeodes sp. could be identified as $P$. charrua Nieto \& Emmerich, 2009 from Uruguay. However, for confirmation of this species, more specimens should be obtained.

\section{Waltzoyphius fasciatus McCafferty \& Lugo-Ortiz, 1995}

Previous distribution. BRAZIL: Amazon Basin (AM, PA), Araguaia-Tocantins Basin (MT, PA), Atlântico Leste Basin (BA), Atlântico Nordeste Oriental Basin (PA), Atlântico Sudeste Basin (ES, RJ, MG), Paraná River Basin (SP), and São Francisco River Basin (GO, MG). COLOMBIA and PARAGUAY.

Material. PERNAMBUCO: Camaragibe, (Rio Besouro, S0759'06.4” W03500'13.9', 66m), 1 nymph, 02/vii/2009, Lima LRC coll. (UFPE); same as preceding except 2 nymphs, 28.II.2010 (UFPE); Igarassu, (Refúgio Charles Darwin, Riacho Jacocá, 748'57.5”S 3457'19.1”W, 37m), 1 nymph, 05.III.2010, Lima LRC coll. (CZNC); Tamandaré, (Reserva
Biológica de Saltinho, Córrego da Sede, $8^{\circ} 43^{\prime} 48.3^{\prime \prime} \mathrm{S} 35^{\circ} 10^{\prime} 35.3^{\prime \prime} \mathrm{W}, 46 \mathrm{~m}$ ), 1 nymph, 21/ix/2009, Lima LRC coll. (UFPE).

\section{Zelusia principalis Lugo-Ortiz \& McCafferty, 1998}

Previous distribution. BRAZIL: Amazon Basin (AM, PA, RR), Araguaia-Tocantins Basin (MT), Atlântico Leste Basin (BA), Atlântico Sudeste Basin (ES, MG, RJ), and Paraná River Basin (MG, SP). COLOMBIA.

Material. PERNAMBUCO: Araçoiaba, (Açude Campo Grande, 750'13.3”S, 356'39.6”W, 133 m), 1 nymph, 09.VII.2009, L.R.C. Lima cols. (UFPE); Rio Formoso, (Fazenda São Manuel, 843'21.51'’S 35¹0'12.65”W, 50m), 4 nymphs, 23.IX.2009, L.R.C. Lima coll. (CZNC); Rio Formoso, (Riacho da Gameleira, Reserva Biológica de Saltinho, 843'12.3"S 35 10 '32.9'W, 47m), 1 nymph, 07.VIII.2010, L.R.C. Lima coll. (UFPE); Tamandaré, (Reserva Biológica de Saltinho, Rio Mamucabas P2, 843'52.7'S $\left.35^{\circ} 10^{\prime 2} 4.8^{\prime \prime W}, 38 \mathrm{~m}\right), 2$ nymphs, 22.IX.2009, L.R.C. Lima coll. (CZNC); Tamandaré, (Reserva Biológica de Saltinho, Córrego da Sede, 843'48.3"S 35¹0’35.3”W, 46m), 2 nymphs, 04.V.2010, L.R.C. Lima coll. (CZNC).

\section{Caenidae \\ Caenis sp.}

Material. PERNAMBUCO: Araçoiaba, (Açude Campo Grande, 7'50'13.3”S 356’39.6”'W, 133m), 1 nymph, 09.VII.2009, L.R.C. Lima coll. (UFPE); Camaragibe, (Rio Besouro, 759'06.4”'S 3500'13.9”'W, 66m), 5 nymphs, 16.VII.2009, L.R.C. Lima coll. (UFPE); Igarassu, (Refúgio Charles Darwin, Riacho Jacocá, 748’57.5”S 345'19.1”W, 37m), 5 nymphs, 22.II.2010, L.R.C. Lima coll. (UFPE); Recife, (Parque Dois Irmãos, $8^{\circ} 00^{\prime} 28.5^{\prime \prime S} 34^{\circ} 56^{\prime} 54.3$ ”'W), $20^{\prime \prime}$ (reared), 12.IX.2009, L.R.C. Lima, G. Nicacio cols. (UFPE); same as preceding except 7 nymphs, 22.VIII.2009 (UFPE); same as preceding except 2 nymphs, $1 O^{\circ}$ (reared), 17.III.2010 (UFPE); Recife, (Riacho da UFRPE - Universidade Federal Rural de Pernambuco, $8^{\circ} 00^{\prime} 57.4^{\prime}$ 'S $34^{\circ} 56^{\prime} 46.4^{\prime \prime} \mathrm{W}, 48 \mathrm{~m}$ ), 4 nymphs, 17.III.2010, L.R.C. Lima coll. (UFPE).

Remarks. Based on nymphs, Caenis sp. could be identified as Caenis pflugfelderi Malzacher, 1990, which was previously recoded from AM (Brazil), but a confirmation of this identification was not possible because male specimens reared had damaged genitalia. However, it appears that forceps in these specimens are apically sharp, while in $C$. pflugfelderi they are rounded. Possibly, in this case, it is a new species, but for confirmation of this species, adult specimens should be obtained from or associated with nymphs.

\section{Leptohyphidae}

\section{Leptohyphes petersi Allen, 1967}

Previous distribution. BRAZIL: Atlântico Leste Basin (BA). PERU.

Material. PERNAMBUCO: Bonito, (Camping Acampamento do Mágico, $8^{\circ} 30^{\prime} 46.8^{\prime \prime}$ S 3543'14”W), 1 nymph, 28.I.2010, L.R.C. Lima; G. Nicacio cols. (UFPE); Bonito, (Cachoeira do Engenho Barra Azul, $8^{\circ} 30^{\prime} 39.3^{\prime \prime S}$ 3543'21.0”'W), 2 nymphs, 28.I.2010, L.R.C. Lima, G. Nicacio cols. (UFPE); Bonito, (Cachoeira Véu da Noiva II, 8`32'51.3”'S 35'43’30.7”W), 1 nymph, 29.I.2010, L.R.C. Lima, G. Nicacio cols (UFPE); São Benedito do Sul,

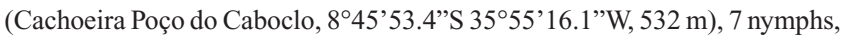
26.II.2011, L.R.C. Lima, F.F. Salles cols. (UFPE). 


\section{Traverhyphes (Mocoihyphes) edmundsi (Allen, 1973)}

Previous distribution. BRAZIL: Araguaia-Tocantins Basin (GO), Atlântico Leste Basin (BA), Atlântico Sul Basin (PR, RS, SC), Paraná River Basin (PR, SP), and Parnaíba River Basin (MA). ARGENTINA.

Material. PERNAMBUCO: São Benedito do Sul, (Cachoeira Poço do Caboclo, 845'53.4”S 3555'16.1'W, 532m), 2 ơ (light trap), 13.XII.2009, L.R.C. Lima, G. Nicacio cols. (UFPE).

\section{Traverhyphes (Traverhyphes) frevo Lima, Salles \& Pinheiro, 2011}

Previous distribution. BRAZIL: Atlântico Nordeste Oriental Basin (PE)

Material. PERNAMBUCO: Bonito, (Camping Bonito Ecoparque, $\left.8^{\circ} 31^{\prime} 58.6^{\prime \prime S} 35^{\circ} 43^{\prime} 26.3^{\prime \prime} \mathrm{W}\right), 6$ O’ $^{\prime}$ (light trap), 29.I.2010, L.R.C. Lima coll. ( 5 at $\mathrm{CZNC}, 1$ at UFPE).

\section{Traverhyphes (Traverhyphes) pirai Molineri, 2001} (RJ).

Previous distribution. BRAZIL: Atlântico Sudeste Basin

Material. PERNAMBUCO: Camaragibe, (Rio Besouro, 759'06.4"S

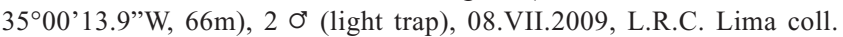
(UFPE); same as preceding except $60^{\prime \prime}$ (light trap), 28.II.2010, L.R.C. Lima coll. (UFPE); Jaqueira, (Rio Pirangi, PE-126 próximo a ponte, $8^{\circ} 44^{\prime} 53^{\prime \prime S}$

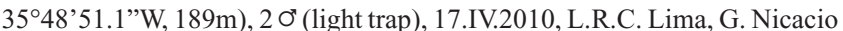
cols. (UFPE); São Benedito do Sul, (Cachoeira Poço do Caboclo,

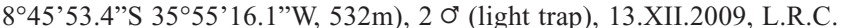
Lima, G. Nicacio cols. (UFPE); Tamandaré, (Reserva Biológica de Saltinho, Córrego da Sede, 843’48.3”S 35¹0’35.3”W, 46m), 3 O' (light trap), 21.IX.2009, L.R.C. Lima coll. (UFPE)

\section{Tricorythodes mirca Molineri, 2002}

Previous distribution. BRAZIL: Atlântico Nordeste Ocidental (MA), Atlântico Sudeste Basin (ES), and Parnaíba River Basin (PI). BOLIVIA.

Material. PERNAMBUCO: Vitória de Santo Antão, (Fazenda Esperança, $\left.8^{\circ} 5^{\prime} 59.51^{\prime \prime S} 35^{\circ} 17^{\prime} 49.6^{\prime \prime} \mathrm{W}\right), 10$ nymphs, 09.VIII.2009, L.R.C. Lima, G. Nicacio, R.P. Salomão (UFPE); same as preceding except 4 nymphs, 30.VIII.2009 (UFPE)

\section{Tricorythopsis spongicola Lima, Salles \& Pinheiro, 2011}

Previous distribution. BRAZIL: Atlântico Nordeste Ocidental Basin (MA), Atlântico Nordeste Oriental Basin (PE), and Atlântico Sudeste (ES).

Material. PERNAMBUCO: Cortês, (Banho da Ilha, 8³0'06.5"S, 35'31'28.6”'W, 223m), 11 nymphs, 20.III.2011, L.R.C. Lima, G. Nicacio cols. (UFPE); same as preceding except 4 nymphs, 05.II.2011; Jaqueira, (Rio Pirangi, PE-126 próximo a ponte, $8^{\circ} 44^{\prime} 53.0^{\prime \prime} \mathrm{S} 35^{\circ} 48^{\prime} 51.1$ 'W, $189 \mathrm{~m}$ ), 9 nymphs, 23.II.2010, L.R.C. Lima coll. (seven at CZNC, two at UFPE); same as preceding except 7 O' (light trap), 17.IV.2010 (CZNC); Ribeirão,
(Rio Amaraji próximo ao Engenho Bastiões, 8³0'49.9"'S 35²4'02.2"W, 114m), 2 nymphs, 05.II.2011, L.R.C. Lima, G. Nicacio cols. (UFPE).

\section{Leptophlebiidae}

Farrodes tepui Domínguez, Molineri \& Peters, 1996 (Figs. 2-5)

Previous distribution. VENEZUELA.

Material. PERNAMBUCO: Bonito, (Rio Verde, Camping Acampamento do Mágico, 8³0'46.8”S 3543'14”'W), 16 Ơ (light trap), 28.I.2010, L.R.C. Lima, G. Nicacio cols. (CZNC); Camaragibe, (Rio Besouro, 759’06.4”S 3500'13.9”'W, 66m), 2 O' (light trap), 02.VII.2009, L.R.C. Lima coll. (CZNC); same as preceding except $5 \mathrm{O}^{\prime \prime}$ (light trap), 08.VII.2009 (CZNC); Jaqueira, (Rio Pirangi, PE-126 próximo a ponte, $8^{\circ} 44^{\prime} 53.0^{\prime \prime}$ 'S $35^{\circ} 48^{\prime} 51.1^{\prime \prime} \mathrm{W}$, 189m), 9 o" (light trap), 17/IV/2010, L.R.C. Lima, G. Nicacio cols. (UFPE); Tamandaré, (Reserva Biológica de Saltinho, Córrego da Sede, S8 43'48.3", W35 $5^{\circ} 0^{\prime} 35.3^{\prime \prime}, 46 \mathrm{~m}$ ), $160^{\prime \prime}$ (light trap), 23.IX.2009, L.R.C. Lima coll. (CZNC); same as preceding except 6 O' (light trap), 16.XI.2009 (UFPE); same as preceding except $10^{\text {( }}$ (reared), 04.V.2010 (CZNC); same as preceding except $2 \sigma^{\circ}$ (reared), 08.VIII.2010 (CZNC); Vitória de Santo Antão, (Bica do Oiteiro, $8^{\circ} 4^{\prime} 42.6^{\prime \prime S} 35^{\circ} 18^{\prime} 16.4 \mathrm{~W}$ ), $10^{\prime \prime}$ (reared), 30.VIII.2009, L.R.C. Lima, G. Nicacio, R.P. Salomão cols. (UFPE).

Remarks. First record of this species from Brazil.

\section{Fittkaulus cururuensis Savage, 1986}

Previous distribution. Brazil: Amazon Basin (PA), Araguaia-Tocantins Basin (MT), and Atlântico Sudeste Basin (ES).

Material. PERNAMBUCO: Igarassu, (Refúgio Charles Darwin, Riacho Jacocá 748'57.5”'S 3457’19.1”W, 37m), 2 nymphs, 22.I.2010, L.R.C. Lima coll. (UFPE); same as preceding except 2 nymphs, 22.II.2010 (UFPE); same as preceding except 2 nymphs, 05/III/2010 (UFPE); same as preceding except 1 nymph, 22.III.2010 (CZNC); São Benedito do Sul, (Cachoeira Poço do Caboclo, $8^{\circ} 45^{\prime} 53.4^{\prime \prime S} 35^{\circ} 55^{\prime} 16.1$ 'W, 532m), 1 nymph, 26.II.2011, L.R.C. Lima, F.F. Salles cols. (UFPE)

Remarks. Due to lack of adult specimens, this species identification was based only by the abdominal color pattern of a mature female nymph.

\section{Hermanella froehlichi Ferreira \& Domínguez, 1992}

Previous distribution. BRAZIL: Atlântico Nordeste Oriental Basin (PE), Atlântico Sudeste Basin (ES), and Paraná River Basin (SP).

Material. PERNAMBUCO: Amaraji, (Rio Amaraji, 8²1'48.9"S 35²8'49.0"W, 320m), 2 O' (reared), 1 ㅇ (reared), 11 nymphs, 20.I.2011, L.R.C. Lima, G. Nicacio cols. (UFPE); Amaraji, (Cachoeira do Rio Morto, $8^{\circ} 26^{\prime} 34.1$ 'S 35²4’22.1'W, 363m), 5 nymphs, 20.i.2011, L.R.C. Lima coll. (UFPE).

\section{Hermanella maculipennis (Ulmer, 1920)}

Previous distribution. BRAZIL: Atlântico Nordeste Oriental Basin (PE), Atlântico Sul Basin (SC), and Paraná River Basin (PR). ARGENTINA. 


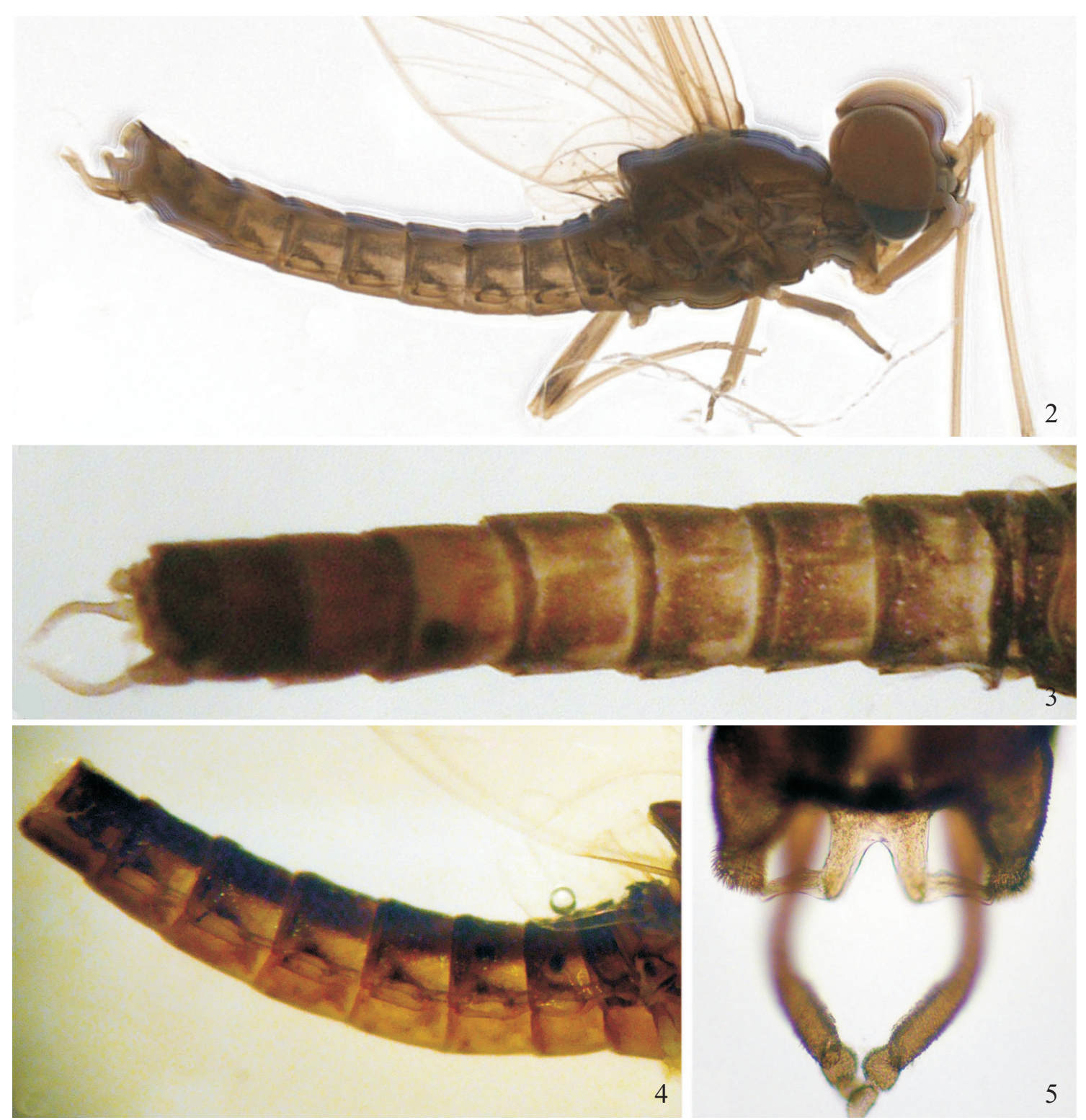

Figs. 2-5. Farrodes tepui Domínguez, Molineri \& Peters, 1996. 2, male imago 1.v.; 3, abdomen d.v.; 4, abdomen 1.v; 5, Genitalia v.v. (d.v. dorsal view; 1.v. lateral view; v.v. ventral view).

Material. PERNAMBUCO: São Benedito do Sul, (Cachoeira Poço do Caboclo, $8^{\circ} 45^{\prime} 53.4^{\prime \prime}$ S $35^{\circ} 55^{\prime} 16.1$ 'W, 532m), 3 nymphs, 28.VIII.2010, L.R.C. Lima col. (UFPE); same as preceding except 1 nymph, 26.II.2011, L.R.C. Lima, F.F. Salles cols. (UFPE); São Benedito do Sul, (Cachoeira

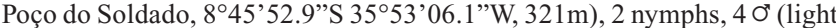
trap), 12.XII.2009, LRC Lima col. (CZNC).

\section{Hydrosmilodon gilliesae Thomas e Péru, 2004}

Previous distribution. BRAZIL: Atlântico Nordeste Oriental Basin (PE); Atlântico Sudeste Basin (ES). FRENCH GUIANA.

Material. PERNAMBUCO: Amaraji, (Rio Amaraji próximo a Usina Amaraji, 8²1'48.9'’S, 35²8’49.0”'W, 320m), 2 nymphs, 20.I.2011, L.R.C. Lima, G. Nicacio cols. (UFPE); Amaraji, (Cachoeira do Rio Morto, $8^{\circ} 26^{\prime} 34.1^{\prime \prime} \mathrm{S} 35^{\circ} 24^{\prime} 22.1^{\prime \prime} \mathrm{W}, 363 \mathrm{~m}$ ), one nymph, 20.I.2011, L.R.C. Lima coll. (UFPE); Bonito, (Rio Verde, Camping Bonito Ecoparque, $8^{\circ} 31$ '58.6”'S 354'ㄹ' 26.3”W), 14 nymphs, 28.I.2010, L.R.C. Lima coll. (UFPE); Cabo de Santo Agostinho, (Rio Pavão, $8^{\circ} 17^{\prime} 51.5^{\prime \prime}$ S 3503'57.1'W, 58m), two nymphs, 18.VIII.2010, L.R.C. Lima coll. (UFPE); Jaqueira, (Rio Pirangi, PE-126 próximo a ponte $8^{\circ} 44^{\prime} 53.0^{\prime \prime} \mathrm{S} 35^{\circ} 48^{\prime} 51.1$ 'W, 189m), 13 nymphs, 12.XII.2009, L.R.C. Lima coll. (UFPE); same as preceding except 6 nymphs, 23.II.2010 (UFPE); same as preceding except 3 nymphs, 17.IV.2010 (UFPE); Ribeirão, (Cachoeira do Amor, 8²6'34.1'S 35²4'22.1'W, 124m), 1 nymph, 21.I.2011, L.R.C. Lima, G. Nicacio cols. (UFPE); São Benedito do Sul, (Cachoeira Poço do Caboclo, 845'53.4"S 35 55'16.1”'W, 532m), 7 nymphs, 13.XII.2009, L.R.C. Lima coll. (UFPE).

\section{Hydrosmilodon plagatus}

\section{Lima, Nascimento \& Salles, 2012}

Previous distribution. BRAZIL: Atlântico Nordeste Oriental Basin (PE); Atlântico Sudeste Basin (ES). 
Material. PERNAMBUCO: Jaqueira, (Rio Piranji, 844'53.0"S $35^{\circ} 48^{\prime} 51.1$ 'W, 189m), 3 nymphs, 12.XII.2009, L.R.C. Lima coll. (UFPE); same as preceding except 2 nymphs, 23.II.2010 (UFPE); same as preceding except 24 nymphs, 1 ơ (light trap), 17.IV./2010 (CZNC).

\section{Leentvaaria palpalis Demoulin, 1966}

Previous distribution. BRAZIL: Atlântico Nordeste Oriental Basin (PE). SURINAME.

Material. PERNAMBUCO: Jaqueira, (Rio Piranji, 844'53.0”S 3548'51.1'W, 189m), 1 nymph, 12.XII.2009, L.R.C. Lima coll. (UFPE); same as preceding except one nymph, 23.II.2010; same as preceding except 2 nymphs, 17.IV.2010 (UFPE); Ribeirão, (Cachoeira do Amor, $8^{\circ} 26^{\prime} 34.1$ 'S 35²4'22.1'W, 124m), 11 nymphs, 21.I.2011, L.R.C. Lima, G. Nicacio cols. (UFPE).

\section{Lisetta ernsti Thomas \& Dominique, 2005}

(Figs. 6-12)

Previous distribution. FRENCH GUIANA.

Material. PERNAMBUCO: São Benedito do Sul, (Cachoeira Poço do Caboclo, $8^{\circ} 45^{\prime} 53.4^{\prime \prime}$ S 35 55'16.1'W, 532m), 1 nymph, 13.XII.2009, L.R.C. Lima coll. (UFPE).

Remarks. First record of the genus and consequently of the species from Brazil.

\section{Miroculis (Miroculis) fittkaui Savage \& Peters, 1983}

Previous distribution. BRAZIL: Amazon Basin (PA) and Atlântico Sudeste Basin(ES). SURINAME and VENEZUELA.

Material. PERNAMBUCO: Camaragibe, (Rio Besouro 759'06.4”S 3500'13.9'W, 66m), 1 nymph, 08.VII.2009, L.R.C. Lima coll. (CZNC); same as preceding except 3 nymphs, 1 ㅇ (reared), 08.IX.2009 (UFPE); same as preceding except 1 nymph, 28.II.2010 (CZNC); Rio Formoso, (Riacho da Gameleira, Reserva Biológica de Saltinho, 843'12.3"S 35¹0'32.9'W, 47m), 6 nymphs, 24.IX.2009, L.R.C. Lima Coll. (UFPE); same as preceding except 1 nymph, 07.VIII.2010 (UFPE); Tamandaré, (Reserva Biológica de Saltinho, Córrego da Sede S843'48.3", W35 10'35.3", 46m), 3 nymphs, 16.XI.2009, L.R.C. Lima coll. (CZNC); same as preceding except 1 \% (reared), 13.V.2010 (CZNC); same as preceding except 1 nymph, 2 ơ (reared), 8 \% (reared), 08.VIII.2010 (CZNC).

\section{Miroculis sp. Savage \& Peters, 1983}

Material. PERNAMBUCO: Amaraji, (Rio Amaraji próximo a Usina Amaraji, 8²1'48.9”'S 35²8'49.0”'W, 320m), 1 O' (light trap), 20.I.2011, L.R.C. Lima (UFPE); same as preceding except $10^{\prime \prime}$ (light trap), 21.IV.2012, L.R.C. Lima (UFPE); same as preceding except $20^{\prime \prime}$ (light trap), 28.IV.2012, L.R.C. Lima (UFPE).

Remarks. Using the key to male imagos proposed by Domínguez et al. (2006), Miroculis sp., would key out in couplet 7(6), which includes Miroculis (Miroculis) marauiae Savage \& Peters, 1983 and Miroculis (Miroculis) rossi Edmunds, 1963. Nevertheless, Miroculis sp. differs from $M$. (M.) rossi by the size of penes $(0.8 \mathrm{x}$ the length of forceps segment I in Miroculis sp., 2.0-2.2 in M. (M.) rossi), as well as the number of facets in male compound eyes (14 facets in Miroculis sp., 6 facets in M. (M.) rossi). Regarding M. (M.) marauiae, the shape of penes (not apically hooked in Miroculis sp.) and posteromedian margin of styliger plate (excavated medially in Miroculis sp.) clearly distinguishes them. It probably represents an undescribed species.

\section{Needhamella ehrhadti (Ulmer, 1920)}

Previous distribution. Brazil: Atlântico Nordeste Oriental Basin (PE); Atlântico Sudeste Basin (RJ), Atlântico Sul Basin (RS), Paraná River Basin (GO, PR), and Uruguai River Basin (SC, RS). ARGENTINA and URUGUAY.

Material. PERNAMBUCO: Amaraji, (Rio Amaraji, 8²1'48.9'S, $35^{\circ} 28^{\prime} 49.0$ "W, 320m), 3 O' (light trap), 20.I.2011, L.R.C. Lima, G. Nicacio cols. (UFPE); Amaraji, (Cachoeira do Rio Morto, 8²6’34.1”S, $35^{\circ} 24^{\prime} 22.1^{\prime \prime} \mathrm{W}, 363 \mathrm{~m}$ ), 1 nymph, 20.I.2011, L.R.C. Lima coll. (UFPE); Jaqueira, (Rio Pirangi, PE-126 próximo a ponte, 844'53.0”S 3548'51.1'W, $189 \mathrm{~m}), 1$ O' (light trap), 12.XII.2009, L.R.C. Lima (UFPE); same as pre-

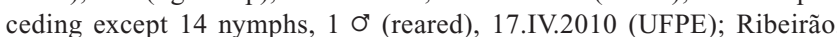
(Cachoeira do Amor, 8²6’34.1”S 35²4’22.1”W, 124m), 1 nymph, 21.I.2011, L.R.C. Lima, G. Nicacio cols. (UFPE); same as preceding except 4 O' (light trap), 05.II.2011 (UFPE); São Benedito do Sul, (Cachoeira Poço do Caboclo, 845'53.4"S 35 55'16.1”W, $532 \mathrm{~m}$ ), 30 nymphs, 26.II.2011, L.R.C. Lima, F.F. Salles cols. (UFPE); São Benedito do Sul,

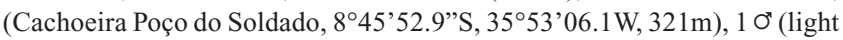
trap), 12.XII.2009, L.R.C. Lima col. (CZNC).

\section{Simothraulopsis (Maculognathus) sabalo Kluge, 2007}

$$
\text { (Figs. 13-18) }
$$

Previous distribution. PERU.

Material. PERNAMBUCO: Camaragibe, (Rio Besouro, 759'06.4'S $\left.35^{\circ} 00^{\prime} 13.9^{\prime} \mathrm{W}, 66 \mathrm{~m}\right), 2$ nymph, 08.IX.2009, L.R.C. Lima coll. (UFPE); Jaqueira, (Rio Pirangi, PE-126 próximo a ponte, $8^{\circ} 44^{\prime} 53.0^{\prime \prime} \mathrm{S} 35^{\circ} 48^{\prime} 51.1^{\prime \prime} \mathrm{W}$, 189m), 5 Ơ (light trap), 12.XII.2009, L.R.C. Lima coll. (CZNC); São Benedito do Sul, (Cachoeira Poço do Caboclo, 845'53.4'S 3555'16.1'”, $532 \mathrm{~m}$ ), 5 nymphs, 5 ơ (light trap), 13.XII.2009, L.R.C. Lima coll. (UFPE); same as preceding 4 nymphs, 26.II.2011, L.R.C. Lima, F.F. Salles cols. (UFPE); Tamandaré, (Reserva Biológica de Saltinho, Rio Mamucabas, $8^{\circ} 43^{\prime 2} 21.6^{\prime \prime} \mathrm{S} 35^{\circ} 11^{\prime} 14^{\prime \prime} \mathrm{W}, 90 \mathrm{~m}$ ), $20^{\prime \prime}$ (light trap), 08.VIII.2010, L.R.C. Lima coll. (UFPE); Tamandaré, (Reserva Biológica de Saltinho, Córrego da Sede,

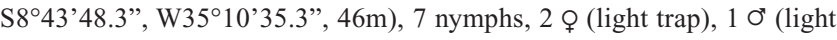
trap), 04.V.2010, L.R.C. Lima coll. (UFPE); Amaraji, (Rio Amaraji próximo a Usina Amaraji, $8^{\circ} 21^{\prime} 48.9^{\prime \prime S} 35^{\circ} 28^{\prime} 49.0^{\prime \prime} \mathrm{W}, 320 \mathrm{~m}$ ), 6 nymphs, $60^{\prime \prime}$ (light trap), 20.I.2011, L.R.C. Lima, G. Nicacio cols. (UFPE).

Remarks. First record from Brazil.

\section{Simothraulopsis janae Mariano, 2010}

Previous distribution. BRAZIL: São Francisco River Ba$\sin (\mathrm{BA})$.

Material. PERNAMBUCO: Amaraji, (Rio Amaraji próximo a Usina Amaraji, 8'21'48.9'S 35²8'49.0”W, 320m), 12 O' (light trap), 20.I.2011, L.R.C. Lima, G. Nicacio cols. (UFPE); Ribeirão, (Rio Amaraji, Cachoeira do Amor, $8^{\circ} 26^{\prime} 34.1^{\prime \prime S} 35^{\circ} 24^{\prime} 22.1$ ”'W, 124m), 6 O' (light trap), 05.II.2011, L.R.C. Lima, G. Nicacio cols. (UFPE). 

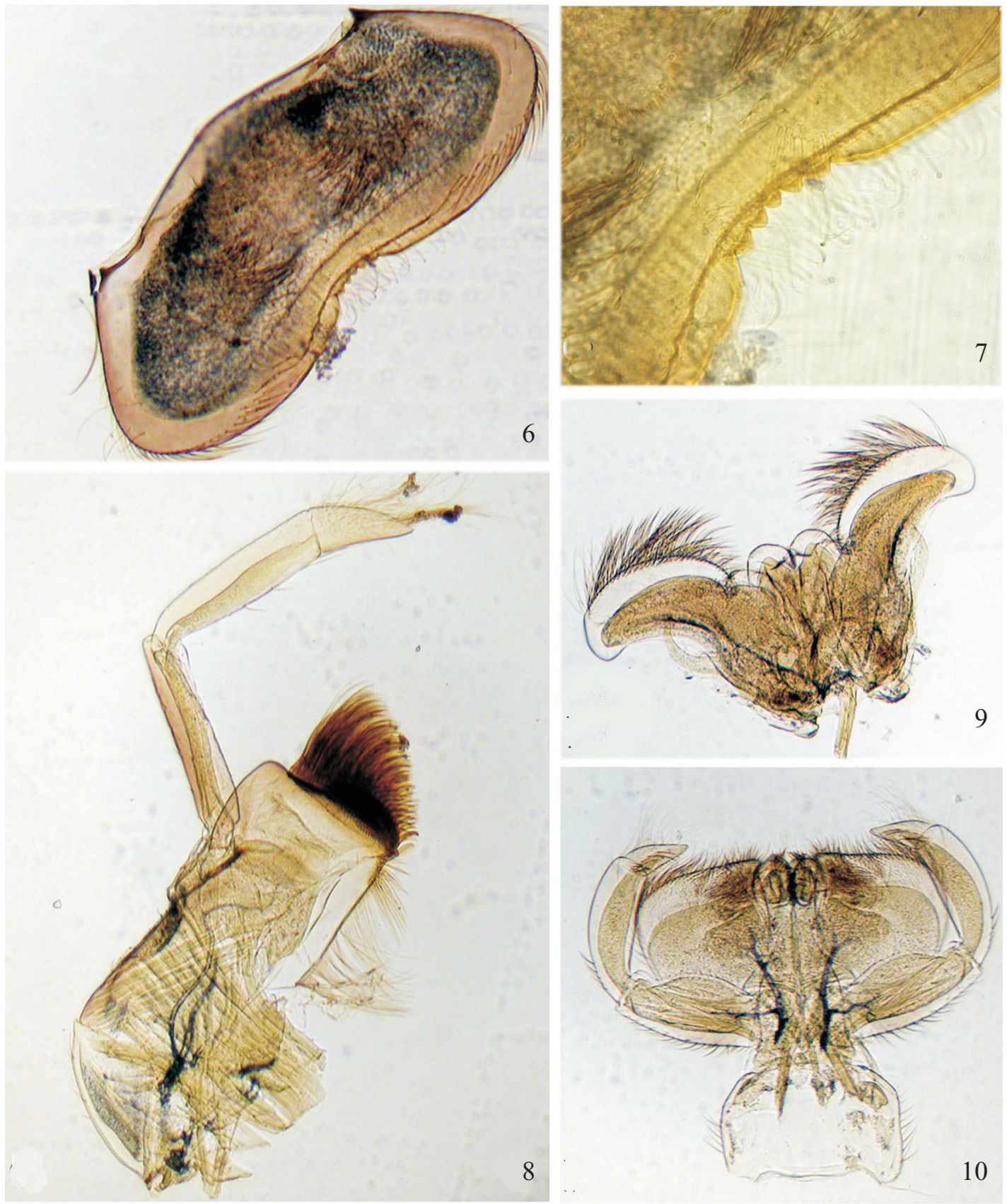

8
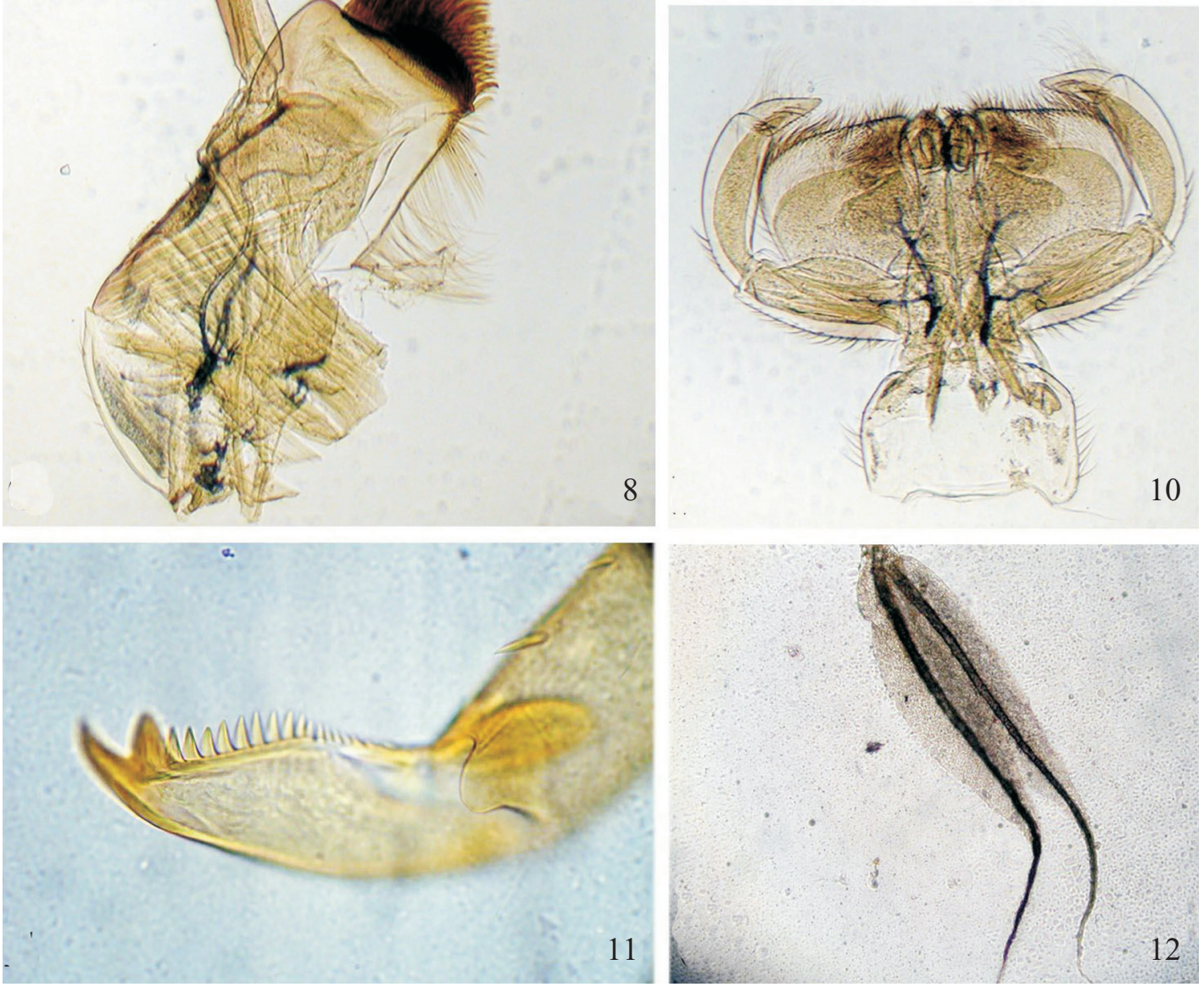

Figs. 6-12. Lisetta ernsti Thomas \& Dominique, 2005. 6, labrum; 7, anterior margin of labrum; 8, maxilla; 9, hipopharynx; 10, labium; 11, tarsal claw I; 12, gill IV. 

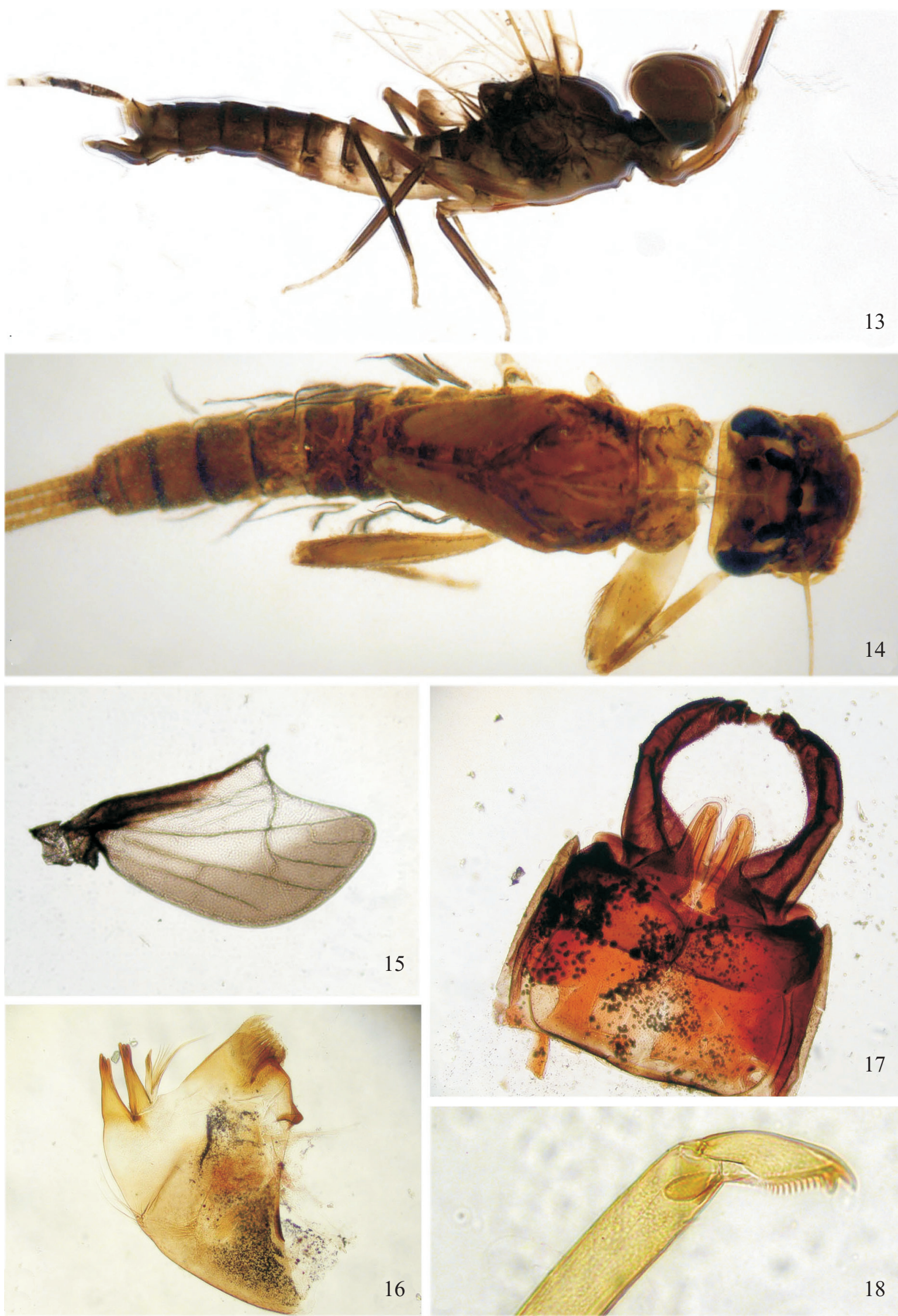

Figs. 13-18. Simothraulopsis (Maculognathus) sabalo Kluge, 2007. 13, male imago 1.v.; 14, nymph d.v.; 15, hind wing; 16, left mandible; 17, genitalia v.v.; 18, tarsal claw I (d.v. dorsal view; v.l. lateral view; v.v. ventral view). 


\section{Terpides sooretamae Boldrini \& Salles, 2009}

Previous distribution. BRAZIL: Araguaia-Tocantins Basin (MT) and Atlântico Sudeste Basin (ES).

Material. PERNAMBUCO: Bonito, (Rio Verde, Camping Bonito Ecoparque, $8^{\circ} 31^{\prime} 58.6^{\prime \prime S} 35^{\circ} 43^{\prime} 26.3^{\prime \prime} \mathrm{W}$ ), 4 nymphs, $1 \mathrm{~F}$ (reared), $1 \mathrm{M}$ (reared), 27.I.2010, L.R.C. Lima coll. (UFPE); Bonito, (Rio Verde, Camping Acampamento do Mágico, 8³0'46.8”S 3543'14”W), 3 nymphs, 28.I.2010, L.R.C. Lima, G. Nicacio cols. (UFPE); São Benedito do Sul, (Cachoeira Poço do Caboclo, 845'53.4”'S 35 55'16.1”W, 532m), 2 nymphs, 2 M (reared), 2 F (reared), 26.II.2011, L.R.C. Lima, F.F. Salles cols. (UFPE).

\section{Polymitarcyidae Campsurus duplicatus Spieth, 1943}

Previous distribution. BRAZIL: Amazon Basin (AM).

Material. PERNAMBUCO: Amaraji, (Rio Amaraji próximo a Usina

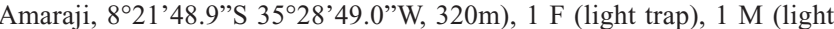
trap), 20.I.2011, L.R.C. Lima, G. Nicacio cols. (UFPE).

\section{ACKNOWLEDGEMENTS}

The authors thank to the staff from Reserva Biológica de Saltinho for logistic support; ICMBio (Instituto Chico Mendes de Conservação da Biodiversidade) and IBAMA (Instituto Brasileiro do Meio Ambiente e dos Recursos Naturais Renováveis) for collecting permits (number 19701); FACEPE (Fundação de Amparo à Ciência e Tecnologia do Estado de Pernambuco) for scholarship and financial support; and CNPq (Conselho Nacional de Desenvolvimento Científico e Tecnológico) for fellowship to FFS.

\section{REFERENCES}

Boldrini, R.; P. V. Cruz; F. F. Salles; E. L. Belmont \& N. Hamada. 2012. Baetidae (Insecta: Ephemeroptera) from northeastern Brazil. Check List 8: 88-94.
Carvalho, A. X. de 2008. Plano Estratégico de Recursos Hídricos e Saneamento. Recife, Secretaria de Recursos Hídricos/Governo do Estado Pernambuco, $452 \mathrm{p}$.

Cruz, P. V.; E. L. Belmont; R. Boldrini \& N. Hamada. 2011. Leptohyphidae (Insecta: Ephemeroptera) from Northeastern Brazil. Neotropical Entomology 40: 682-688

Dias, L. G.; F. F. Salles \& P. S. Ferreira. 2008. New species of Tricorythopsis Traver (Ephemeroptera: Leptohyphidae) from northern Brazil. Studies on Neotropical Fauna and Environment 43: 237-241.

Domínguez, E.; C. Molineri; M. L. Pescador; M. D. Hubbard \& C. Nieto. 2006. Ephemeroptera of South America. Aquatic Biodiversity in Latin America (ABLA) Volume 2. Sofia-Moscow, Pensoft, 646 p.

Lima, L. R. C.; F. F. Salles; U. S. Pinheiro \& E. Quinto. 2010. Espécies de Baetidae (Ephemeroptera) do Sul da Bahia, com descrição de uma nova espécie de Paracloeodes day. Neotropical Entomology 39: 725-731.

Lima, L. R. C.; F. F. Salles \& U. S. Pinheiro. 2011. New species of Leptohyphidae (Ephemeroptera) from northeastern Brazil. Zootaxa 3050: 63-68.

Lima, L. R. C.; J. M. C. Nascimento; R. MarianO; U. Pinheiro \& F. F. Salles. 2012. New species and new records of Hermanella complex (Ephemeroptera: Leptophlebiidae) from Eastern Brazilian Coast. Annales de Limnologie - International Journal of Limnology 48: 201-213.

Mariano, R. 2010. Two new species of Simothraulopsis Traver, 1947 (Ephemeroptera: Leptophlebiidae: Atalophlebiinae) from northeastern Brazil. Aquatic Insects 32: 129-134.

Salles, F. F. 2006. A ordem Ephemeroptera no Brasil (Insecta): taxonomia e diversidade. Thesis, Universidade Federal de Viçosa, $300 \mathrm{p}$

Salles, F. F; R. Boldrini; J. C. M. Nascimento; E. A. Raimundi \& Y. F. Shimano. 2012. Lista de espécies de Ephemeroptera (Insecta) registradas para o Brasil. Available at: http://sites.google.com/site/ ephemeropterabr/(acessed 9 May 2012).

Salles, F. F.; E. R. Da-Silva; M. D. Hubbard \& J. E. Serrão. 2004. As espécies de Ephemeroptera (Insecta) registradas para o Brasil. Biota Neotropica 4: 1-34

Salles, F. F.; E. A. Raimundi; R. Boldrini \& G. M. Souza-Franco. 2010. The genus Americabaetis Kluge (Ephemeroptera: Baetidae) in Brazil: new species, stage description, and key to nymphs. Zootaxa 2560: 16-28.

Thomas, A.; Y. Dominique \& V. Horeau. 2001. Les Ephémères de La Guyane Française. 7. Redescription de Camelobaetidius cayumba (Traver \& Edmunds, 1968) [Ephemeroptera, Baetidae]. Ephemera 3: 117-122.

Traver, J. R. \& G. F. Edmunds Jr. 1968. A revision of the Baetidae with patulateclawed nymphs (Ephemeroptera). Pacific Insects 10: 629-677.

Received 1/11/2011; accepted 18/7/2012

Editor: Daniela Maeda Takiya 